\title{
« Water: spatial dynamics, competitive claims and governance. Reducing stress on the resource in urban, peri-urban and rural areas »
}

\section{Compte rendu d'atelier scientifique (Pondichéry, Inde, 30 novembre - $1^{\mathrm{er}}$ décembre 2007)}

\author{
Olivia Aubriot ${ }^{1}$, Olivier Petit $^{2}$ \\ 1 Agroethnologue, UPR 299, Milieux, sociétés et cultures en Himalaya, CNRS, 7 rue Guy-Môquet, BP 8, 94801 Villejuif cedex, France \\ 2 Économiste, EA 4026, EREIA, Université d'Artois, 9 rue du Temple, BP 10665, 62030 Arras cedex, France
}

Cet atelier international, organisé conjointement par K.V. Devi Prasad, du département d'écologie et des sciences de l'environnement de l'Université de Pondichéry, O. Aubriot, de l'Institut français de Pondichéry (IFP), et S. Janakarajan, de l'Institut d'études du développement de Madras (MIDS, Chennai), a réuni 25 présentations orales et posters de chercheurs indiens et étrangers (notamment français), écologues, géographes, sociologues, anthropologues et économistes, ainsi que quelques représentants d'ONG indiennes.

L'atelier a débuté par une séance plénière pendant laquelle un chercheur indien, membre du Groupe intergouvernemental sur l'évolution du climat (GIEC), a discuté les enjeux des changements climatiques, notamment pour le sous-continent indien. Une présentation a également porté sur une synthèse des questions principales abordées dans cet atelier, ce qui a permis de faire ressortir certains des enjeux transversaux aux espaces ruraux, périurbains et urbains et de justifier les trois notions abordées dans le titre même de l'atelier. Ainsi, la gouvernance des ressources en eau vise à résoudre les conflits générés par la concurrence entre les usages, sur des territoires pertinents, qu'ils soient naturels ou artificiels. Les présentations ont ensuite été réparties sur trois sessions, aux thèmes suivants : dynamique spatiale d'utilisation de

Auteur correspondant : Olivia Aubriot, oaubriot@vjf.cnrs.fr De 2005 à 2007, O. Aubriot était en poste à l'Institut français de Pondichéry pour diriger, au sein du département Sciences sociales, le programme de recherche Gestion sociale de l'eau. l'eau; compétition entre usages de l'eau en zone urbaine et périurbaine ; unités spatiales et sociales de gestion de l'eau. Les questions quantitatives relatives à la pollution ont par ailleurs été regroupées en une session de trois posters. Lors de la première soirée, l'IFP a saisi l'occasion de cet atelier pour lancer officiellement le DVD trilingue Eaux douces, eaux amères, réalisé par Nathanaël Coste et Nicolas Ploumpidis, deux anciens étudiants en master de géographie de l'Université Paris 1 Panthéon-Sorbonne.

L'objectif premier de cet atelier était d'amener les participants à aborder les questions de gestion de l'eau dans leur dimension spatiale. Car, si la prise en compte de cette dimension est assez naturelle pour les chercheurs français, elle ne l'est pas pour la majorité de leurs homologues indiens, qui ne considèrent pas l'aspect spatial comme problématique et qui ne verbalisent donc pas les situations en ces termes. Notons, toutefois, que ce n'est pas l'absence de support cartographique qui permet d'expliquer cet écart d'approche, puisque la plupart des travaux se sont appuyés sur des cartes, des produits de systèmes d'information géographique (SIG), voire de télédétection, pour illustrer les propos. Cette façon d'aborder les questions, sans mettre la dimension spatiale au cœur de la réflexion, est peut-être liée au faible développement en Inde de la géographie, discipline peu populaire, mal connue et dénigrée par de nombreux autres chercheurs de sciences sociales.

De l'ensemble des thématiques abordées et à l'issue des discussions, on peut retenir plusieurs idées transversales intéressantes. 
1. Le bassin n'est pas l'unité de gestion appropriée à toutes les situations.

Plusieurs présentations ont tenté de faire apparaître, cartes à l'appui, la variété des territoires de l'eau : territoires «naturels » des bassins versants, systèmes aquifères, dérivations techniques (canaux, tanks, isolés ou en interconnexion), territoires de gestion. Les questions d'extraterritorialité de l'eau (du bassin versant au bassin déversant, comme l'on dit parfois, ou du watershed au water-shared, comme l'a souligné une des intervenantes) permettent d'expliquer finalement bien des conflits d'usage sur ces ressources.

2. La dimension spatiale de la compétition pour l'eau de surface ne doit pas être limitée à la concurrence amontaval.

La concurrence amont-aval est récurrente dans tout système gravitaire, que ce soit à l'échelle d'un fleuve, de larges systèmes d'irrigation ou d'un canal de distribution de l'eau. En raison des progrès techniques qui permettent de stocker davantage d'eau et en raison des politiques de développement qui incitent à ne pas "gaspiller» l'eau en la laissant s'écouler vers la mer, chaque lieu du bassin, notamment en amont, s'approprie le maximum possible de la ressource, ce qui rend les conflits amontaval encore plus vifs. Toutefois, la compétition pour l'eau ne peut plus être analysée uniquement dans cette optique amont-aval. En effet, de nombreux acteurs, qui rendent facilement responsables de leurs difficultés ceux qui sont en amont, devraient aussi mettre en cause l'utilisation de l'eau au sein de leur propre unité administrative ou de gestion, et considérer les disparités spatiales et les dysfonctionnements locaux. S'en tenir à une optique amont-aval minimise finalement les changements dans les choix politiques et les modes de gestion de la ressource (cas de l'Andhra Pradesh et du Tamil Nadu, respectivement en aval de la Krishna et de la Cauvéry).

3. Se surajoute aux compétitions entre les divers secteurs économiques de l'eau (domestique, agricole, industriel, urbain), celle entre ces secteurs et les besoins environnementaux.

La présentation de plusieurs cas du sud de l'Inde (dans le bassin du fleuve Krishna, mais aussi au Tamil Nadu et dans l'État de Karnataka) a souligné la nécessité, à côté des usages anthropiques de l'eau, de conserver les ressources pour assurer la capacité de renouvellement des écosystèmes. Cette dernière question apparaît de manière particulière dans le bassin du fleuve Krishna (qui couvre plus de $250000 \mathrm{~km}^{2}$ ), où les dérivations successives pour les besoins humains «ferment» le bassin, peu d'eau s'écoulant dorénavant vers la mer. L'écosystème des zones côtières s'en trouve transformé. De plus, cela entraîne de sérieuses répercussions socioéconomiques pour tous les habitants qui dépendent, pour leur activité professionnelle, de l'utilisation de l'eau. Des questions sur la valeur écologique de l'eau, l'intérêt et les limites de sa prise en compte ont alors émergé.

4. Un développement urbain, et parfois industriel, dépendant de ressources hydriques lointaines crée de nouvelles situations conflictuelles.

Plusieurs présentations se sont intéressées à l'alimentation en eau de villes indiennes (Bangalore, Chennai, Tirupur). Les projets choisis par les municipalités sont systématiquement coûteux et dépendants de ressources hydriques lointaines. Certains chercheurs (mais, de façon assez surprenante, pas tous) ont remis en cause l'absence de traitement et de recyclage de l'eau - choix qui permettraient pourtant de dépendre davantage des ressources locales - et la préférence pour des projets qui créent des situations conflictuelles avec les zones d'extraction de l'eau (zones périurbaines ou rurales). Le cas de la ville de Chennai est en cela exemplaire par la désertification agricole de la zone périurbaine en raison de l'envoi vers la capitale régionale de l'eau pompée dans les nappes souterraines. Celui de la zone de Tirupur est également impressionnant par la quasi-absence de traitement des eaux, qui conduit à une forte dégradation de leur qualité, et par la concurrence économique entre agences d'alimentation en eau de la ville.

5. Les thèmes des droits de propriété et des grands principes des politiques de l'eau sont à approfondir.

Les questions sur la gouvernance des ressources en eau ont également été abordées par les intervenants, en lien avec la problématique des droits de propriété. Par exemple, l'un des participants a montré que les lois sur l'eau sont trop vagues pour que les personnes puissent se défendre individuellement en justice. Plusieurs communications ont choisi de revenir sur les grands principes des politiques de l'eau, tels qu'ils sont définis à l'échelle internationale (par le Conseil mondial de l'eau, le Partenariat mondial de l'eau, etc.). Mais en l'absence de perspective critique à l'égard de ces grands principes (la dimension locale participative, la gestion par bassins, la mobilisation des outils économiques, la gestion intégrée des ressources en eau), la confusion est souvent de mise, en Inde comme ailleurs. Or, comme l'ont souligné plusieurs intervenants, le caractère polysémique de ces grands principes (par exemple, que signifie précisément la gestion participative? Qui participe concrètement? À quoi les acteurs participent-ils?), leur flou partagé, doivent être l'occasion d'un travail scientifique approfondi, visant à en souligner les présupposés, les incohérences et le caractère trop souvent normatif. En effet, les injonctions des bailleurs de fonds internationaux (relayées par les politiques publiques nationales) à se conformer à ces principes, sans que soient prises en compte les particularités géographiques, culturelles et politiques à l'échelle locale, sont rarement analysées dans une perspective critique. Mais ce travail ne peut être réalisé que dans une démarche interdisciplinaire 
assumée, tant il est vrai que les dimensions multiples de la gestion de l'eau ne peuvent pas être appréhendées correctement par des disciplines isolées.

6. Quelle unité sociale de gestion : la communauté villageoise ou des institutions ad hoc?

Dans un contexte général de pénurie d'eau, le partage de la ressource devrait reposer sur des formes de gouvernance fondées sur la participation des acteurs et sur le respect du principe d'équité entre les usagers, ce qui, dans une société encore fortement hiérarchisée, reste de l'ordre $\mathrm{du}$ discours. Une des intervenantes a rappelé toutefois que la communauté est souvent perçue par de nombreux agents de développement comme un idéal égalitaire et de cohésion sociale; or, concrètement sur le terrain, cette vision se vérifie rarement, il faut donc s'en prémunir. Pourtant, tous les membres d'ONG qui ont présenté leur action prennent comme unité sociale de gestion de l'eau l'échelle villageoise, certains prenant toutefois garde de distinguer les différents acteurs et leurs intérêts diversifiés. D'autres sont confrontés au problème de changement d'échelle de leur approche afin de toucher davantage de villages ou de régions. Les ONG, qui détiennent une place active dans le processus de participation dans la gestion des ressources naturelles, se positionnent souvent comme intermédiaire entre l'État, inefficace ou peu réactif aux problèmes quotidiens des villageois, et les villageois eux-mêmes, selon le principe de subsidiarité. La communication d'un intervenant, portant sur l'un des programmes du gouvernement du Tamil Nadu pour la réhabilitation des infrastructures villageoises traditionnelles (les tanks ou étangs-réservoirs) sans intervention d'ONG, a montré à quel point les institutions ad hoc créées par des ingénieurs, hors des réalités sociales, génèrent pour l'instant davantage de situations conflictuelles que de cohésion sociale dans la gestion de ces étangs. Si la participation peut se révéler relativement active pour la gestion des ressources communes traditionnelles (étangs), elle ne l'est guère (voire n'est même pas envisagée dans de nombreux programmes) pour les eaux souterraines, dont l'exploitation développe l'individualisme des exploitants.

En définitive, le spectre très large des questions traitées au cours de cet atelier, la diversité des perspectives disciplinaires ayant pu s'exprimer et la grande variété des cas d'étude présentés offrent une image d'un champ de recherche en questionnement perpétuel. Si l'un des objectifs, convaincre les chercheurs indiens présents de la nécessité d'une prise en compte de la dimension territoriale, n'a pas été entièrement rempli, gageons que l'atelier a toutefois suscité chez eux un intérêt pour cette démarche et qu'ils poursuivront la réflexion dans ce sens sur leurs propres terrains. La publication d'une sélection d'articles, suite à cet atelier, devrait les inciter à intégrer ces paramètres. 